\title{
Infantile Intraoral Myofibroma of the Gum in A 2-Year-Old Child
}

\author{
Martin Igaz ${ }^{1}$, Martin Kopáni² and Csaba Biró ${ }^{3 *}$ \\ ${ }^{1}$ Laboratorium Dr. Kosak und Partner, Mariannengasse 14/ 20 G u. 3OG, TOP 3-5, 1090 Wien, Austria \\ ${ }^{2}$ Institute of Medical Physics, Biophysics, Informatics and Telemedicine Medical Faculty of Comenius University, Bratislava, Slovakia \\ ${ }^{3}$ Department of Pathology Institute of Oncology St. Elizabeth, Bratislava, Slovakia
}

\begin{abstract}
Intraoral myofibroma is a rare tumor. It occurs most frequently in childhood, in particular between the ages of 2 and 9, mostly intraosseally in the mandibula, tongue, oral mucosa and less frequently in the gum. Clinical assessment of the lesion due to rapid growth may imitate malignancy. Histomorphologically, the myofibroma is mostly a benign lesion with excellent prognosis. A sufficient surgical excision is a sufficient therapy. The most common alternatives in the differential diagnostics include fibrous epulis, pyogenic granuloma, peripheral odontogenic fibroma, nodular fasciitis, fibroma of tendon sheaths, leiomyoma, dermatomyofibroma, myoepricytoma, glomangiopericytoma, angioleiomyoma. Results of genetic examinations reveal a vascular disease with impairment of pericytes or their progenitors that are connected with point mutations of $5 \mathrm{q} 32$ gene. In our case, we present an examination for consultation on an intraosseous tumor in a 2-year-old boy with minimum clinical history, accessible perioperative local finding of the $4 \times 3 \mathrm{~cm}$ tumor in the mandibular area with usuration of the bone, with only several torn, greyish to white pieces sent for examination.
\end{abstract}

Keywords: Intraoral Infantile Myofibroma, PDGFR1, Receptor Tyrosine Kinases, Myofibroblast

\section{Introduction}

Myofibroma is a benign superficial neoplasm with perivascular myoid differentiation. ${ }^{[1]}$ It may be solitary (myofibroma) or multiple (myofibromatosis). Myofibroma / myofibromatosis occurs at all ages, most frequently in childhood between the age of 2 to 9 , more frequently in boys. As far as anatomic distribution is concerned, it is most often (58\%) located in the head and neck area, followed by lower extremities (14\%), trunk (13\%), upper extremities $(10 \%)$ and internal organs (1\%). In addition, there are reports of cases with anatomic location in the oral cavity: in the mandibula, maxilla, lip area, facial mucosa, tongue as polypoid intraoral projection, in the palatal area, base of the mouth. ${ }^{[2]}$ Multicentricity is reported in $4 \%$ of cases, mainly with familial occurrence, occurring more frequently in girls. The inheritance pattern is autosomally dominant. Histology reveals that superficial soft tissues of the skin, subcutaneous tissue, bones, bone marrow and parenchymatous organs (heart, lungs, liver) are mostly affected. Intraosseal occurrence is more common in children with no occurrence in adults. The behavior of myofibroma / myofibromatosis is mostly benign. There have been fatal cases with several focal organ impairment of the heart, lungs, liver. Solitary myofibroma may recur in 7 to $13 \%$ with spontaneous regression of the tumor in most cases. Multifocal nodules with visceral lesions have a worse prognosis. ${ }^{[1]}$.

\section{Case Report}

In our case, the examination was performed for consultation purposes. At the time of the examination, we had little knowledge of the patient and only very brief information. We knew that an intraoral tumor in a 2-year-old boy is involved, with accessible perioperative local finding of a $4 \times 3 \mathrm{~cm}$ tumor in the area of the mandibula with bone usuration, with only several torn, greyish to white pieces sent for examination. Tumor samples were fixed in $10 \%$ buffered formalin and embedded in paraffin. Histological slices were studied following staining with hematoxylin and eosin and 10 antibodies based on methods of a standard immunohistochemical technique. Information on the antibodies with pretreatment and incubation is shown in Table 1. For visualization, we used EnVision DAKO: K5007 and Bond Polymer Refine DS9800 Leica Biosystems. Histomorphologically, a histoid proliferation of fusiform cells was involved. Some were cigar-shaped, others literally thin and wavy, thinker, thinner, at some places with an indication of alternating regular intervals of proliferated fusiform cells (Fig 1) and production of whirpool structures with smaller, rather oval and round cells with more eosinophilic cytoplasm, in serial cuts also with a discrete alternation with the fusiform cell component (Fig 2-3). The interstitium was fibrotizing and at certain places even hyalinized, with the presence of capillaries and vessels and an indication of plexiform 
architecture. Focally, proliferated connective cells showed atypia. Mitotic activity was 3-4 mitoses per 10 high power field (HPF). Connective cells with no position, so-called cell to cell. Small bone lamellas were only sporadically proliferated and mostly occurred in serial cuts on the periphery on the lesion surface. There were no necroses. At some places on the periphery of proliferation, there were pieces of surrounding striated muscles and fat tissue with local infiltration of the proliferation. It was not our aim to prove vascular propagation. Immunohistochemically, keratin cocktail (AE1/AE3) negative, intermediate filament for mesenchymal tissue (vimentin) positive, actin, alpha smooth muscle type (SMA) positive, $53 \mathrm{kDa}$ intermediate filament (desmin) negative, high molecular weight caldesmon (H-caldesmon) negative, acidic protein (S100) negative, platelet endothelial cell adhesion molecule (CD31) positive in vessels, marker of hematopoietic progenitor cells and endothelial cells (CD34) positive in vessels, macrosialin (CD68) focally slightly positive, marker of cell proliferation (Ki67) negative reaction.

Table 1: Antibody information.

\begin{tabular}{|c|c|c|c|c|}
\hline Antibody & Source & Pretreatment & Dilution & Incubation (min.) \\
\hline AE1/AE3 & Dako & Citrat, pH6 & $1: 100$ & 30 \\
Vimentin & Dako & Citrat, pH6 & $1: 150$ & 30 \\
Aktin (ASM) & Dako & Tris-EDTA,pH9 & $1: 420$ & 30 \\
Desmin & Novocastra & Tris-EDTA,pH9 & $1: 80$ & 30 \\
H-Caldesmon & Dako & Tris-EDTA,pH9 & $1: 370$ & 30 \\
S100 protein & Dako & Tris-EDTA,pH9 & $1: 80$ & 30 \\
CD34 Class II & Dako & Tris-EDTA,pH9 & RTU & 30 \\
CD31 & Dako & Tris-EDTA,pH9 & $1: 100$ & 30 \\
CD68(PG-M1) & Dako & Tris-EDTA,pH9 & $1: 120$ & 30 \\
Ki67 & Cako & Citrat, pH6 & \\
\hline
\end{tabular}

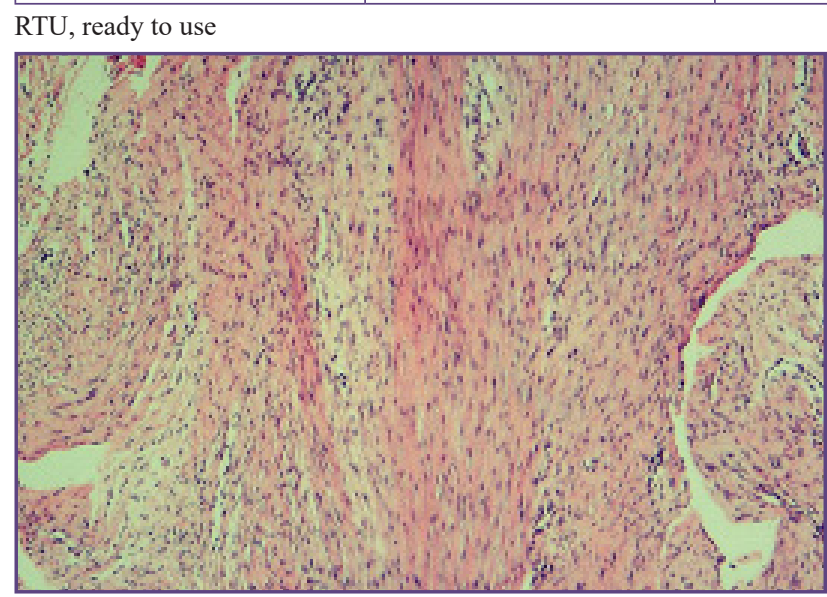

Fig. 1: Proliferation of spindle, round, ovoid and plump cells, H\&E, 100x.

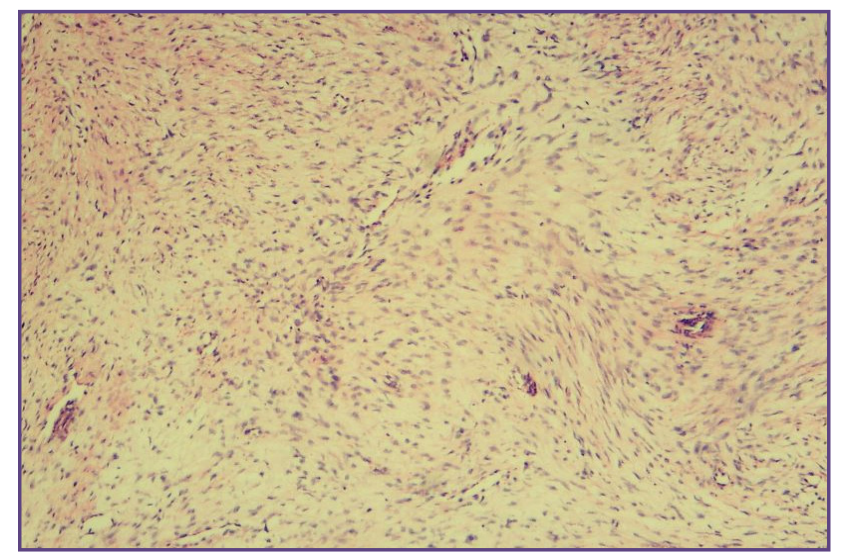

Fig. 2: Biphasic component with myoid nodes, H\&E, 100x.

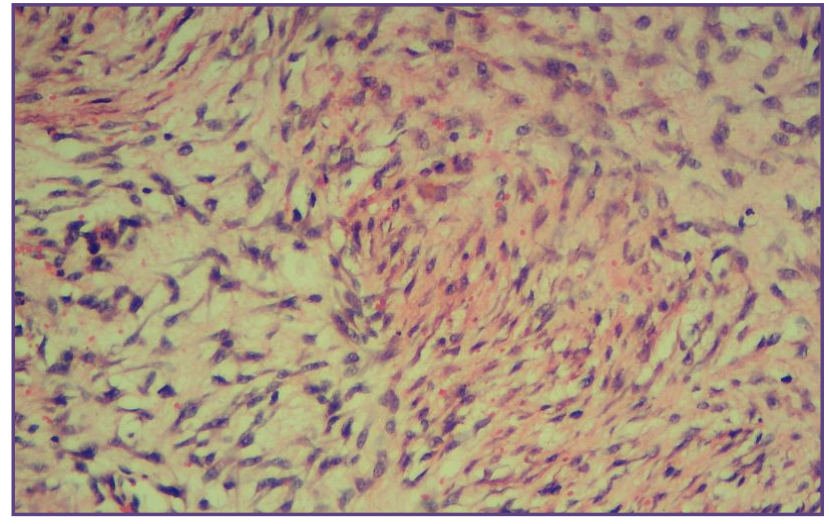

Fig. 3: Biphasic component with myoid nodule, H\&E, 200x 


\section{Discussion}

In addition to myofibroma, the group of perivascular myoma neoplasm includes myopericytoma, glomangiopericytoma, angioleiomyoma, the histomorphological picture of which is very similar and therefore their differentiation is arbitrary nowadays, depending on the location, patient age and clinical manifestation. Myofibroma, myopericytoma, glomus tumor and angioleiomyma are nowadays morphologically classified as a single development line. ${ }^{[1]}$ Macroscopically, it is a solitary or multiple painless tumor with average size from 1 to $5 \mathrm{~cm}$, often with infiltrating edges. Skin lesions are hyperemic, imitating the vascular tumor; subcutaneous lesions move freely and do not hurt; deeper lesions or lesions occurring in organs are fixed and cause impairment of the organ with clinical manifestations. [2] Histomorphologically, it is a biphasic tumor with pseudocapsular boundary on the periphery, of multinodular appearance. Proliferation consists of two cell components: one component is primitive, comprising small round and oval or ovoid fusiform cells with a small cytoplasm, associated with multiple blood capillaries with thin walls, relatively often with central coagulation necroses as well, less frequently with calcifications, hyalinization, cystic degeneration; the other component comprises spirallike nodes and clumps of plump fusiform cells with slim nuclei and pale eosinophilic cytoplasm. These two components are represented in tumor proliferation either in variable manner, or randomly, more frequently with zonal distribution: the primitive component in the centre and spiral-like myoid nodes on the periphery. Reverse zoning with myoid nodes in the centre may be also present. Myoid nodes are of basophil, pseudochondroid appearance, mostly with prominent stromal hyalinization or calcifications that may be dominant. Rarely, the neoplasm may have high cellularity, with infiltrative growth pattern, which however is not an indication of a more aggressive behavior of the tumor and must not be mistaken for malignity. ${ }^{[1]}$ Mitoses are rare (up to 5 mitoses per $10 \mathrm{HPF}$ at the most). High cellularity lesions may contain as many as 8 mitoses per $10 \mathrm{HPF}$. Myoid nodes typically bulge into thin-wall vessels in the form of subendothelial formations. ${ }^{[2]}$ Immunohistochemically, the biphasic tumor proliferation is positive for vimentin, SMA, $\mathrm{H}$ - caldesmon, focally may be positive for desmin $(+/-)$, but is mostly negative. It is negative for keratin cocktail, S100, CD31 (positive vessels), CD34 (positive vessels). ${ }^{[3]}$ Recently, myofibromas have been the subject of genetic studies as well. Such studies involved in particular familial myofibromas. It is assumed that similar mutations may be also present in solitary myofibromas without familial occurrence, although the formation of solitary myofibromas has not been sufficiently explained until now.
${ }^{[4]}$ In term of genetics, hereditary cases involve germinal point mutations of the type $\beta$ platelet-derived growth factor receptor (PDGFR - BETA, PDGFRB, PDGFR1). The gene is located on the chromosome 5q32. It encodes the tyrosine kinase transmembrane receptor protein PDGFRB. PDGFRB is physiologically expressed in fibroblasts, pericytes. ${ }^{[4]}$ Point mutations occur in particular in the region of the transmembrane domain, kinase domain, in particular in the juxtamedullary domain and tyrosine kinase domain with amine lobe (N-lobe) and sporadically with carboxyl terminal lobe (C-lobe). Most often identified have been the following point PDGFRB mutations: somatic mutation of p.N666K, germinal point mutations of p.P660T, p.R561C, two-hit mutations: germinal mutation (p.R561C) associated with somatic mutation (p.N666K) and cis somatic mutations: mutations located on the same allele: p.W566R / p.N666K (cis). Complete activation of the PDGRB gene requires both germinal and somatic or cis somatic mutation. After germinal mutations of the gene, PDGFRB behaves as oncogene. ${ }^{[4]}$ As part of the differential diagnostics, the fibroepithelial polyp is associated with chronic inflammatory cellulisation of the oral and gum mucosa which often calcifies or ossifies; it is most frequently diagnosed as fibrous epulis, pyogenic granuloma and peripheral odontogenic fibroma. Connective cells are positive for vimentin and S100-negative.$^{[5]}$ Nodular fasciitis is a reactive proliferation of fibroblasts and myofibroblasts in myxoid stroma with prominent vasculature (with granulation tissue-like vascular proliferation), lymphocytes, fresh bleeding, typical zoning - hypercellular periphery and hypocellular center, located in particular in subcutis and on fascias, mainly rapid-growing, with maximum size of 2-3 cm. Immunohistochemically with SMA positivity, muscle specific actin (MSA, HHF35), vimentin, actin filament associated regulatory protein (calponin), CD68. [6] Fibroma of tendon sheaths (tendosynovial fibroma) occurs in older patients, in particular in fingers, hands, wrists, with size up to $3 \mathrm{~cm}$ and often overlapping with a picture of nodular fasciitis. ${ }^{[2]}$ Leiomyoma creates nodes, clumps of fine fusiform cells with eosinophilic cytoplasm, blunt fusiform nuclei, fine chromatin, with no mitoses, no necroses, positivity for SMA, desmin, H-caldesmon and CD34-negativity. ${ }^{[7]}$ Dermatomyofibroma is a plaquelike skin lesion occurring mostly in adults and only rarely in children, with positivity for SMA, vimentin and CD34negativity. ${ }^{[2]}$ Myopericytoma and angioleiomyoma belong to the group of perivascular myoid tumors with positivity for SMA, desmin, H-caldesmon, but without a proof of mutations in the myofibroma. Glomangiopericytoma is another member of the group of perivascular myoid tumors, in particular in the region of the nail bed, with hemangiopericytoid vessels and fusiform glomus cells, 
with positivity for SMA, vimentin, CD34, H-caldesmon, and negativity for desmin, S100. ${ }^{[1]}$

\section{Conclusion}

Based on patient age, tumor location, histomorphological picture, results of immunochistochemistry tests and as part of the differential diagnosis, excluding other lesions, the tumor proliferation was diagnosed as infantile intraoral myofibroma of the gum.

\section{Competing Interests}

The authors declare that they have no conflict of interests.

\section{Reference}

1. Hornick JL. Practical Soft Tissue Pathology: A Diagnostic Approach Pattern Recognition Series 2nd Ed., Elsevier 2018.

2. Miettinen M. Benign fibroblastic and myofibroblastic proliferations in children. In: Miettinen M. Modern soft tissue pathology: tumors and non-neoplastic conditions, Cambridge University Press; 2010, 273 - 279.

3. Sundaravel S., Anuthama K., Prasad H. et.al. Intraosseous myofibroma of mandible: A rarity of jaws: With clinical, radiological, histopatological and immunohistochemical features. J Oral Maxillofac Pathol. 2013 Jan - Apr; 17(1):121-125.

4. Arts F.A. et al. PDGFRB gain-of-function mutations in sporadic infantile myofibromatosis. Human Molecular Genetics, 2017, Vol. 26, No. 10, 1801-1810.

5. Ajura AJ., Lau SH. Gingival myofibroma in childern: report of 4 cases with immunohistochemical findings. Malaysian J Pathol. 2007; 29(1) : 53 - 56.

6. Nirvikalpa N., Narayanan V. Intraosseous infantile myofibroma off the mandible. Annalas of Maxillofacial Surgery, January - June 2011. Volume 1. Issue 1.p. 87 - 90.

7. Kim KW. et al. Solitary myofibroma of the adult mandible: A case report and review of literature. Korean Journal of Pathology 2014; 48: 307-310.

*Corresponding author:

Csaba Biró, Department of Pathology Institute of Oncology St. Elizabeth, Bratislava, Slovakia

Phone: +91 421904948473

Email: csaba.biro1675@gmail.com

Financial or other Competing Interests: None. 